\section{Biomimetización de carillas directas con resina compuesta y restauraciones indirectas con silicato de circonio}

\author{
Biomimicry of direct veneers with compound resin and indirect \\ restaurations with zirconium silicate
}

\begin{abstract}
Resumen
Actualmente contamos con diversos materiales para realizar restauraciones directas en el sector anterior. En el presente caso se utilizó una resina directa, que es un compuesto nanohíbrido que tiene características ópticas de los dientes naturales. De igual forma para las restauraciones indirectas existen materiales que reproducen las características ópticas similares a los tejidos dentarios, en el caso se utilizó una resina indirecta de laboratorio, que es un silicato de circonio que combina las ventajas de la cerámica y la resina. En el presente caso clínico se detalla el protocolo de la aplicación de la resina directa y la resina indirecta. Resultados: El resultado obtenido colmó las expectativas del paciente ya que se pudo restaurar las piezas dentarias involucradas devolviendo la función perdida y sobre todo la estética de una manera adecuada biomimetizando ambos materiales. Conclusiones: El conocimiento profundo de ambos materiales a utilizar así como las técnicas y protocolos a seguir, llevaron a biomimetizar los resultados estéticos de la zona anterior obteniendo translucidez, opacidad y opalescencia muy semejante en todos los dientes restaurados.
\end{abstract}

Palabras clave: Resina directa; resina indirecta; carillas; biomimetización.

\section{Abstract}

Now we have various materials to for direct restorations in the previous sector. In this case the direct resin was used Beautiful II, which is a nanohybrid compound with optical characteristics of natural teeth. Likewise for indirect restorations exists materials which reproduce the optical characteristics similar to the dental tissues, in this case indirect laboratory resin Ceramage was used, which is a zirconium silicate that combine the advantages of ceramic and resin.

In the present clinic case it's detailed the protocol of the application of the resin Beautiful II and Ceramage. Results: the result high patient expectations because it was possible to restore the teeth involved restoring function loss and especially aesthetics in a suitable manner biomimicking both materials. Conclusions: deep knowledge of both materials to be used as well as the protocols and techniques to follow, led to biomimic the aesthetic results of the previous zone getting translucency, opacity and opalescence very similar in all restored teeth.

Keywords: Direct resin; indirect resin; veneers; Biomimicry.

\section{Introducción}

La evolución de los sistemas adhesivos y de las resinas compuestas fotopolimerizables ha originado el desarrollo de diversas técnicas estéticas restauradoras, con el objetivo de reparar alteraciones de color, forma o posición dental que perjudiquen el equilibrio estético o funcional $^{1}$. Entre estas alternativas está la confección de la carilla directa de resina compuesta, que consiste en la aplicación y estratificado de una o más capas de este material sobre la superficie del diente, para favorecer, principalmente un resultado estético ${ }^{1-2}$. sina compuesta permiten al profesional
La realización de carillas directas de re- controlar y evaluar el procedimiento restaurador, desde la selección del color hasta el establecimiento de la morfología final. Este tipo de restauración generalmente puede confeccionarse en una sola sesión clínica. Por requerir poco o incluso ningún desgaste del diente, la carilla directa de resina compuesta representa una alternativa restauradora menos invasiva y de menor costo, en comparación con la corona o laminado de porcelana.

Estos factores, aliados a una buena relación costo-beneficio, tanto para el paciente como para el profesional, hacen que la realización de esta técnica restauradora sea cada vez más popular, pues es posible mejorar la estética dental rá-
Caso Clínico
Ernesto Vílchez-Salazar ${ }^{1}$, Alberto Flores-Huamaní ${ }^{2}$, Ernesto Vílchez-Quintana ${ }^{3}$, Carol Torres-Roque ${ }^{3}$, Marisabel Vílchez-Quintana ${ }^{4}$, Henry Reynoso-Vílchez ${ }^{5}$
1.Departamento Académico de Estomatología Rehabilitadora de la Facultad de Odontología de la Universidad Nacional Mayor de San Marcos, Perú.

2.Facultad de Odontología de la Universidad San Martin de Porres, Perú.

3.Escuela de Pregrado de la Facultad de Odontología de la Universidad Nacional Mayor de San Marcos, Perú.

4.Escuela de Pregrado de la Facultad de Estomatología de la Universidad Peruana Cayetano Heredia, Perú.

5.International Dental Training Center. MDT. Esp. en Cerámica en USA, Japón, Alemania.

Correspondencia:

Mg. Esp. Ernesto Vilchez Salazar

Universidad Nacional Mayor de San Marcos, Facultad de Odontología. Av. Amezaga 375,

Lima 1, Perú.

Correo electrónico: evilchezsalazar@gmail.com

\section{Coautores:}

Flores : dentalflores@hotmail.com

Vilchez-Quintana E.:

ernestovilchezq@hotmail.com

Torres-Roque : caroltorresroque@gmail.com Vilchez-Quintana M. :

marisabel.vilchez@upch.pe

Reynoso-Vilchez : henry_labdent@hotmail.com

Recibido: 05-04-15

Aceptado: 10-06-15

pidamente. Además, las carillas directas de resina compuesta presentan una expectativa de longevidad clínica en torno a los 8 ańos ${ }^{3}$.

Como en cualquier técnica restauradora adhesiva, la obtención del éxito funcional y estético depende principalmente del dentista, que debe entender los principios básicos de los sistemas adhesivos y de las resinas compuestas, diagnosticar el caso clínico con una visión multidisciplinaria y observar los fundamentos de la estética dental para intentar reproducirla ${ }^{4}$.

Beautifil II de la marca SHOFU, es un compuesto nanohíbrido con tecnología Giomer. Los productos con tecnología 
Giomer a diferencia de los ionómeros de vidrio y compómeros que requieren la absorción de agua después del fotocurado para liberar fluoruro, contienen un núcleo de vidrio multifuncional que se somete a una reacción ácido-base durante la fabricación y es posteriormente protegida por una capa de superficie modificada, esta estructura trilaminar forma una especie de ionómero de vidrio estable que permite la liberación de iones y de recarga, al tiempo que protege el núcleo de vidrio de los efectos dañinos de la humedad, lo que mejora la durabilidad a largo plazo. Los productos con tecnología Giomer son capaces de lograr la absorción de iones de productos para el hogar de higiene dental, tales como pasta de dientes, proporcionando beneficios sostenidos de re mineralización a la estructura de los dientes adyacentes durante la vida de la restauración en boca.

El relleno en la estructura de esta resina ha sido desarrollado para simular la estructura interna de los dientes naturales con la transmisión de luz ideales y las características ópticas. La translucidez moderada y transmisión de la luz del esmalte combinado con la luz-difusión de la dentina ofrece una estética predecible con un partido de sombra cerca de los dientes naturales ${ }^{5,6.7}$.

Otra de las alternativas es realizar una restauración indirecta para lo cual podemos utilizar las resinas indirectas de laboratorio, que son compuestos que combinan las ventajas de la cerámica y la resina. Ceramage de la marca SHO$\mathrm{FU}$, es una resina de laboratorio a base de silicato de circonio integrado para restauraciones indirectas para regiones anteriores y posteriores ${ }^{8,9,10}$.

La transmisión natural de la luz y la fluorescencia de las resinas de laboratorio permiten que las restauraciones exhiban una apariencia natural en la boca del paciente.

Reproduce prácticamente la apariencia y las propiedades de transmitir la luz de la dentina y el esmalte ${ }^{1,11}$.

Una completa selección de componentes (modificadores de color, efectos translúcidos) proporcionan una manera fácil de resolver los desafíos interorales como con porcelana ${ }^{12-13-14}$.

\section{Reporte de caso:}

Paciente de sexo masculino de 45 años de edad que acude a consulta expresando el deseo de realizar su rehabilitación en el sector anteroposterior.

Al examinar al paciente se observó que en la pieza 1.2 necesitaba una corona, ausen- cia de la 22 y las piezas 1.1 y la 2.1 presentaban restauraciones con resinas desbordantes y con cambio de coloración.

\section{Diagnóstico}

Del estado de salud general.- Paciente con aparente buen estado de salud general, sin riesgo sistémico al tratamiento estomatológico.

Del estado de salud bucal.- Paciente edéntulo parcial superior con ausencia de pza. 2.2.

Presencia de poste de fibra de vidrio en pza. 1.2

Presencia de restauraciones desbordantes y discromía en pzas.1.1 y 2.1

\section{Plan de tratamiento}

1. Fisioterapia Oral, enseñanza de la técnica del cepillado, hila dental y enjuague bucal.

2. Restauraciones directas con la resina compuesta Beautifil II.

3. Restauraciones indirectas con las resinas de laboratorio (Ceramage).

\section{Tratamiento:}

Se procedió a realizar la obtención del modelo de diagnóstico.

Luego se procedió a realizar el análisis funcional de las piezas. (Fig 1)

En la pieza 1.2 se talló para corona y se tomó la impresión definitiva con silicona de adición. Se confeccionó una corona con Ceramage de manera estratificada indirectamente. (Fig 3)

Luego se cementó la corona con el cemento Monocem de la Shofu. (Fig 4)

Se procedió a realizar las preparaciones para las carillas directas en los dos centrales para lo cual se colocó hilo retractor \#000 y se procedió a realizar los tallados con una fresa cilíndrica de punta redonda; en la pieza 1.1 se realizó mayor desgaste por presentar una discromía más marcada. En la pieza 2.1 el desgaste fue de $0.5 \mathrm{~mm}$. La preparación a nivel cervical fue yuxtagingival. (Fig 2)

Una vez realizada la preparación vestibular de las piezas procedemos a realizar la aplicación del adhesivo autograbante de sétima generación BeautiBond (SHOFU) durante 10 segundos, luego se secó con aire suavemente durante 3 segundos y finalmente se curó con lámpara de polimerización led por 5 segundos.

Se procedió a realizar la estratificaión de las capas de resina Beautifil II. (Fig 4)

En la pieza 2.2 se confeccionó un puente tipo Maryland con Ceramage.(Fig.4)

\section{Resultados}

Los resultados fueron satisfactorios, ya que se logró devolver la estética y la función del paciente. Como se aprecia en las figuras, la biomimetización obtenida entre ambos materiales: resina directa (Beautifil II) y resina indirecta (Ceramage) fue realmente óptima, logrando observar en todas las restauraciones la misma translucidez, opacidad y opalescencia características de todo diente natural. Fig. 5

\section{Discusión}

Siempre que se va a rehabilitar el sector anterior pensamos en el factor estético, aún más cuando vamos a realizar la rehabilitación con dos tipos de alternativas, como pueden ser una carilla directa con resina compuesta y restauraciones indirectas como en este caso clínico; se debe tener cuidado en la estratificación para ir comprendiendo las dimensiones de los colores como son matiz croma y valor y de esta manera lograremos una biomimetización de los dientes a restaurar que sea imperceptible a la percepción ocular.

Una buena fotopolimerización así como un pulido prolijo de las restauraciones directas garantizarán una estadía duradera de las resinas mayor a 8 ańos, esto también dependiendo de la buena higiene que presente nuestro paciente.

En el caso de las resinas indirectas la longevidad es mayor gracias a su composición y al sistema de polimerizado, las cuales pueden estar en boca según reporta la bibliografía por más de 15 años.

\section{Conclusiones}

Con el descubrimiento de los nuevos materiales restauradores y las nuevas tecnologías, se han ido modificando y acortando los protocolos de tratamiento a utilizar, lo que nos advierte de que se debe estar en continua actualización de los nuevos materiales que van apareciendo.

El manejo adecuado de los materiales y de las técnicas a usar, nos garantizará el éxito de nuestros tratamientos restauradores.

Hoy en día la variedad en gamma de resinas y tonalidades así como también de efectos, nos permite poder imitar al diente natural casi a la perfección, mimetizándolo en boca de manera tal que las restauraciones pasen imperceptibles al ojo humano.

La mimetización de las resinas directas e indirectas, hasta hace algunos años era 


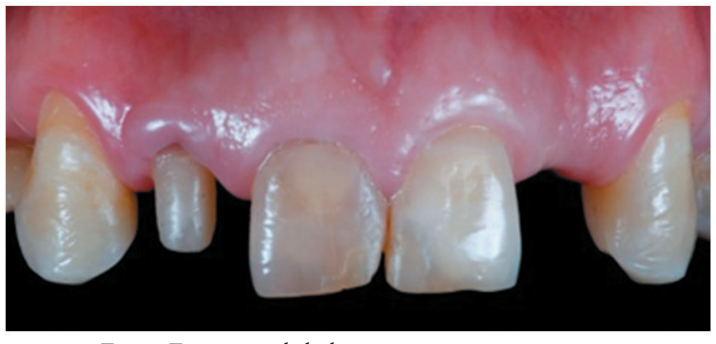

Fig.1 Foto inicial de las piezas a restaurar

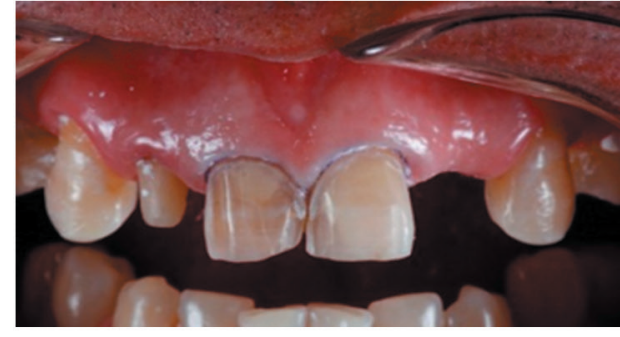

Fig. 2 Preparación de las piezas dentarias
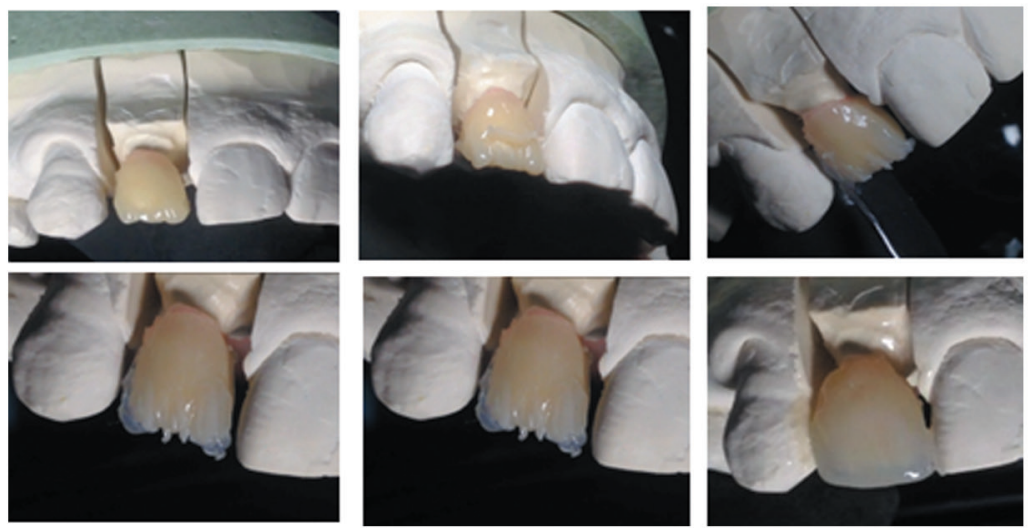

Fig 3. Confección de corona con Ceramage

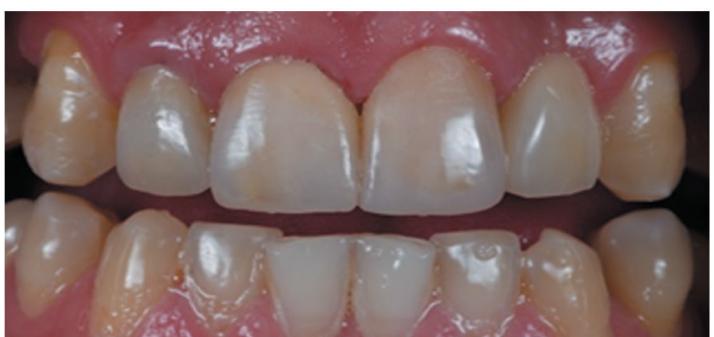

Fig. 4 Estratificación por capas de las piezas restauradas

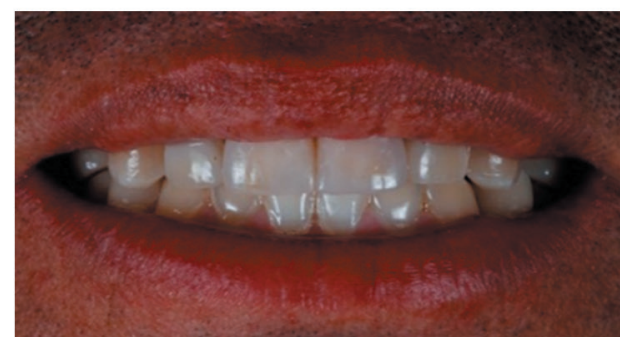

Fig.5 Resultado final del caso cosa poco manejable debido a la escacez de variedad de tonalidades; las resinas Beautifil II con tecnología Giomer y las de Ceramage nos han demostrado en este caso clínico que la biomimetización lograda es tal que pasa invisible a la vista.

\section{Referencias bibliográficas}

1. Hirata R. Claves en Odontología estética. $1^{\mathrm{a}}$ ed. Buenos Aires: Médica Panamericana; 2012. 162-201p.

2. 4Kina S. Restauraciones estéticas cerámicas. $1^{\mathrm{a}}$ ed. Buenos Aires: Médica Panamericana; 2011. 280335 p.

3. Bertoldi A. Rehabilitación post endodóntica. Buenos Aires: Médica Panamericana; 2012. 141-181p.

4. Nocchi E. Odontología restautradora. 2a ed. Buenos Aires: Médica Panamericana; 2008. 160-171p.

5. Toshiyuki Nakatsuka, Shigehisa Inokoshi: Technical innovation of a composite resin world, development and its process of GIOMER; Dental Echo. 2001; 123:2-17

6. Ardu S, Krejci I. Biomimetic direct composite stratification technique for the restoration of anterior teeth. Quintessence Inter 2006,37(3):167-74.

7. Magne P, Holz J. Stratification of composite restorations. Systematic and durable replication of natural aesthetics. Pract Periodont Aesthet Dentistry 1996;8:61-8.

8. Hidalgo RC. Técnica de estratificación por capas naturales a mano alzada con resinas compuestas. Visión Dental 2008;11(3):412-418

9. Lamas C, Angulo G. Reconstrucción del Sector Anterior con Resinas Compuestas, Odontol.San Marquina, 2009;12(2):90-92p.

10. Albers HF. Tooth colored restorations: principles and techniques.9th edition. Ontario, Canadá: $\mathrm{BC}$ Decker publishing. 2002; 203p.

11. Conceicao EN et al. Restauracoes Estéticas. Compósitos, Cerámicas e Implantes. Porto Alegre, Brasil: Artmed; 2005. 152p

12. Soares C, Guimaraes C, Freitas P, Soares A. Effects of previous treatments on bond strength of two selfetching adhesive systems to dental substrate. Journal of adhesive dentistry 2007;9:291-296p.

13. Breschi L, Mazzoni A, Ruggeri A, Cadenaro M, Di Lenarda R, De Stefano Dorigo E. Dental adhesión review: aging and stabilitry of the bonded interface. Dent Mater. 2008; 24(1):90-101p.

14. Hebling J, Pashley DH, Tjaderhane L, Tay FR. Chlorhexidine arrests subclinical degradation of dentin hybrid layers in vivo. J Dent Res. 2006; 85(4):384. 